\title{
Life-course socio-economic factors, skin colour and abdominal obesity in adulthood in a Brazilian birth cohort
}

\author{
David A González ${ }^{1, *}$, Aydin Nazmi², John S Yudkin ${ }^{3}$ and Cesar G Victora ${ }^{1}$ \\ ${ }^{1}$ Post-graduate Program in Epidemiology, Federal University of Pelotas, Brazil, Rua Marechal Deodoro 1160 , \\ Pelotas, RS, CEP 96020-220, Brazil: ${ }^{2}$ Department of Food Science and Nutrition, California Polytechnic State \\ University, San Luis Obispo, CA, USA: ${ }^{3}$ University College London, London, UK
}

Submitted 15 January 2009: Accepted 5 May 2009: First published online 6 August 2009

\begin{abstract}
Objective: Obesity is an increasingly prevalent nutritional disorder throughout the world. In particular, abdominal obesity is associated with cardiovascular and metabolic risk. The present study aimed to evaluate the effects of skin colour and life-course socio-economic indicators on waist circumference (WC), hip circumference (HC) and waist:hip ratio (WHR) in young adults.

Design: Population-based birth cohort study. Individuals born in 1982 in Pelotas (southern Brazil) were visited on a number of occasions from birth to age 23-24 years. A sample of the cohort was sought in 2006 and 972 individuals were located. The analysis was restricted to individuals with complete data available (442 males, 414 females).

Results: In men, family income at birth and in 2004-5 were positively associated with WC and HC, but not with WHR. Regardless of current income, men born to wealthier families had larger WC and HC as adults. Skin colour was not associated with any of the outcomes. In women, early poverty was associated with smaller HC, and current poverty with larger WC. Poverty at any age thus led to higher WHR. Black women had larger WC and HC than white women, but there were no differences in WHR. All the associations were partially mediated by education and behavioural variables.

Conclusions: The effects of early socio-economic position on WC and HC persist even after adjustment for adult socio-economic position, highlighting the importance of interventions during the first years of life.
\end{abstract}

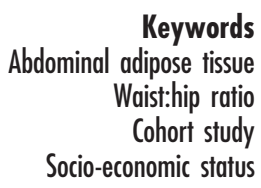

Obesity is a key risk factor for the incidence of and mortality associated with CVD, diabetes, hypertension and hypercholesterolaemia ${ }^{(1-5)}$. Over the past three decades, its prevalence has increased rapidly throughout the world in both sexes ${ }^{(6-8)}$, but men and women are differentially affected according to their socio-economic position (SEP). In middle-income countries, obesity is more prevalent in richer men and poorer women ${ }^{(7,8)}$.

Obesity in adults is usually evaluated by $\mathrm{BMI}^{(3)}$. However, BMI does not provide information on fat distribution, which is a better indicator of CVD risk ${ }^{(9)}$. There have been calls to use waist:hip ratio (WHR) instead of other anthropometric measures for assessing CVD risk ${ }^{(1,2,5)}$. A meta-regression published in 2007 showed that WHR was more strongly associated with CVD than waist circumference (WC) among 4355 incident cases ${ }^{(1)}$. The INTERHEART Study evaluated 12461 cases of acute myocardial infarction in fifty-two countries, confirming these findings even among individuals with normal or low BMI (BMI $\left.<25 \mathrm{~kg} / \mathrm{m}^{2}\right)^{(5)}$. It is important to study WC and hip circumference (HC) separately because the adverse effects of WHR on CVD could be due to either a relatively large $\mathrm{WC}$ or a small $\mathrm{HC}^{(10,11)}$. Furthermore, both measures represent two distinct time periods of growth and may be determined by different factors ${ }^{(12-14)}$.

Nearly all studies evaluating socio-economic risk factors for obesity in adults are cross-sectional ${ }^{(8,15,16)}$. However, there is evidence that early poverty affects nutritional status in adult life $\mathrm{e}^{(17-19)}$. Studies evaluating the long-term effects of early SEP on abdominal obesity in adults are rare and principally limited to data from high-income countries; these report inverse associations in women and variable patterns for men ${ }^{(18,20-27)}$. Research in this area is particularly relevant for low- and

*Corresponding author: Email david.epidemio@gmail.com

(C) The Authors 2009. The online version of this article is published within an Open Access environment subject to the conditions of the Creative Commons Attribution-NonCommercial-ShareAlike licence $<$ http://creativecommons.org/licenses/by-nc-sa/2.5/ $>$. The written permission of Cambridge University Press must be obtained for commercial re-use. 
middle-income settings to better understand the effects of rapid nutritional transition ${ }^{(8)}$.

Studies examining the effects of ethnicity or skin colour have also been exclusive to high-income countries and have shown conflicting results ${ }^{(18,28-30)}$. A recent literature review indicated that variations in body composition between blacks and whites are the result of a complex multifactorial entanglement of lifestyle, environmental and genetic differences ${ }^{(31)}$, but more studies are required to clarify the mechanisms involved.

Considering the limited literature on the long-terms effects of SEP and ethnicity on body composition, the current study evaluated the influence of family income and skin colour on WC, HC, and WHR at age 23-24 years in a population-based birth cohort. Special attention was given to socio-economic trajectories from birth to adulthood and the possible mediating roles of behavioural variables.

\section{Methods}

Pelotas is a southern Brazilian city with a population of 340 000. In 2002 the city had a per capita Gross Domestic Product of \$US 1958 (national mean \$US 2604) ${ }^{(32)}$. The main ethnic groups include European and African descendents, with a small indigenous Brazilian population. The 1982 Pelotas birth cohort study included 5914 liveborn babies ( $99 \cdot 2 \%$ of all births) to mothers who lived in the urban area of the city. Mothers were weighed and interviewed in the hospital and babies were examined after birth and subsequently followed up on numerous occasions from infancy until the age of 23-24 years. Details regarding follow-up visits are shown in Fig. 1 and described elsewhere ${ }^{(33)}$.

In 1997, seventy of the city's 265 census tracts were randomly selected and all households in each sector were visited, leading to 1076 cohort members being located (follow-up rate $72 \%$ ). Between October 2004 and August 2005, the entire cohort was sought through a census of all 98000 urban households (follow-up rate 77\%). Information on socio-economic and health variables was collected. Between January and April 2006, the 1076 cohort members examined in 1997 were sought. Anthropometric, behavioural and health variables were collected on 972 subjects $(90 \cdot 3 \%$ of those studied in 1997). Assessment of the three dependent variables (WC, HC and WHR) was carried out in 856 subjects ( $88.1 \%$; see Table 1). For the measurements, interviewers were trained and standardized. WC and HC were taken with individuals in standing position using a flexible $160 \mathrm{~cm}$ (precision: $1 \mathrm{~mm}$ ) fibreglass measuring tape. The waist was considered the narrowest part of the trunk and was taken directly on the skin. The hip was measured at the widest point of the buttocks while wearing a gown over underwear, in order to protect privacy. A linear regression equation was used to correct the gowned measures (hip $_{\text {corrected }}=-0 \cdot 978+1.002 \times$ gowned measure) based on a validation study undertaken in 105 volunteers (fiftyseven men, forty-eight women). Quality control measures included repeating $10 \%$ of interviews during field work by a supervisor, showing over $95 \%$ repeatability.
FOLLOW-UP

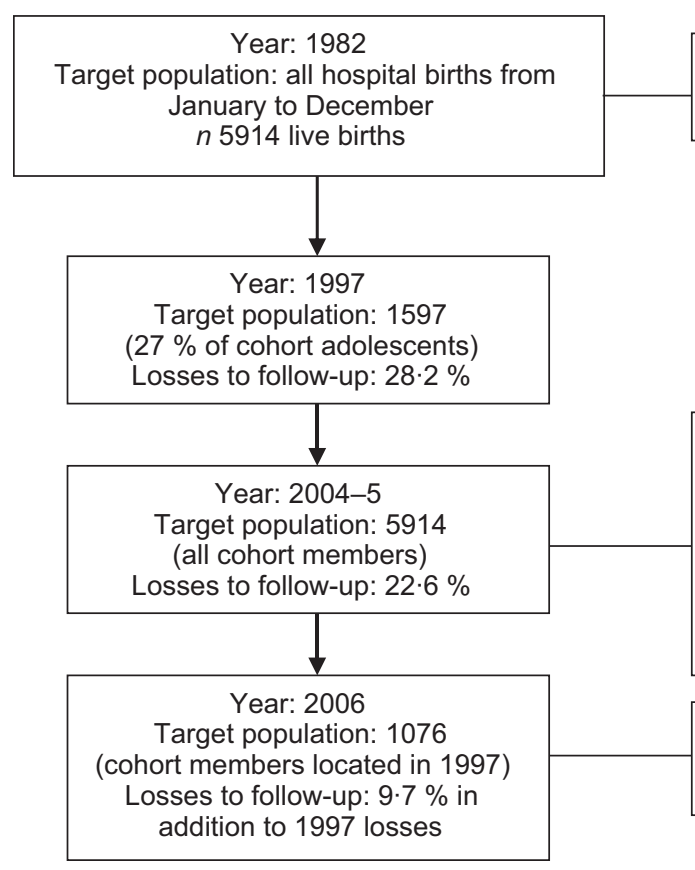

VARIABLES

Family income Maternal education

\section{Family income Own attained education \\ Self-reported skin colour Height, weight and BMI \\ Behavioral variables: physical activity, alcohol consumption, smoking, fibre and fat intake Parity (women)}

Waist circumference Hip circumference Waist:hip ratio

Fig. 1 Follow-up visits and variables used from the 1982 Pelotas birth cohort study 
Table 1 Comparison of demographic and socio-economic characteristics at birth between cohort members measured and not measured in 2006 ( $n$ 856): 1982 Pelotas birth cohort study, Brazil

\begin{tabular}{|c|c|c|c|c|c|c|}
\hline \multirow[b]{3}{*}{ Variables* } & \multicolumn{6}{|c|}{ Cohort members measured in 2006 related to: } \\
\hline & \multicolumn{2}{|c|}{ Original cohort } & \multicolumn{2}{|c|}{ Located in 1997} & \multicolumn{2}{|c|}{ Located in 2006} \\
\hline & $n$ & $\%$ measuredt & $n$ & $\%$ measuredt & $n$ & $\%$ measuredt \\
\hline \multicolumn{7}{|l|}{ Sex } \\
\hline Male & 3037 & $59 \cdot 1$ & 561 & $78 \cdot 9$ & 494 & $89 \cdot 5$ \\
\hline Female & 2876 & $57 \cdot 5$ & 515 & $80 \cdot 4$ & 478 & $86 \cdot 6$ \\
\hline$P$ value $\ddagger$ & & $0 \cdot 5$ & & $0 \cdot 6$ & & $0 \cdot 2$ \\
\hline \multicolumn{7}{|c|}{ Maternal skin colour } \\
\hline White & 4851 & $58 \cdot 9$ & 909 & $79 \cdot 0$ & 824 & $87 \cdot 0$ \\
\hline Other & 1060 & $55 \cdot 8$ & 166 & $83 \cdot 1$ & 147 & 93.9 \\
\hline$P$ value $\ddagger$ & & 0.3 & & 0.2 & & 0.02 \\
\hline \multicolumn{7}{|c|}{ Family income at birth (minimum wages) } \\
\hline$\leq 1$ & 1288 & $48 \cdot 7$ & 182 & $76 \cdot 4$ & 160 & $86 \cdot 9$ \\
\hline $1 \cdot 1-3$ & 2789 & $59 \cdot 2$ & 514 & $80 \cdot 2$ & 456 & $90 \cdot 4$ \\
\hline$>3$ & 1808 & $64 \cdot 1$ & 376 & $80 \cdot 5$ & 352 & $85 \cdot 8$ \\
\hline$P$ value $\ddagger$ & & $<0.001$ & & 0.5 & & $0 \cdot 1$ \\
\hline \multicolumn{7}{|c|}{ Maternal education (years) } \\
\hline $0-4$ & 1960 & $54 \cdot 4$ & 321 & $78 \cdot 8$ & 289 & $87 \cdot 5$ \\
\hline $5-8$ & 2454 & $60 \cdot 7$ & 455 & $81 \cdot 5$ & 412 & $89 \cdot 8$ \\
\hline $9+$ & 1493 & $59 \cdot 6$ & 298 & $77 \cdot 5$ & 269 & $85 \cdot 9$ \\
\hline$P$ value $\ddagger$ & & 0.08 & & 0.4 & & 0.3 \\
\hline \multicolumn{7}{|c|}{ Birth weight $(\mathrm{g})$} \\
\hline$\geq 2500$ & 5375 & $58 \cdot 0$ & 1010 & $80 \cdot 3$ & 914 & $88 \cdot 6$ \\
\hline$<2500$ & 534 & $61 \cdot 9$ & 66 & $69 \cdot 7$ & 58 & $79 \cdot 3$ \\
\hline$P$ value $\ddagger$ & & 0.4 & & 0.04 & & 0.03 \\
\hline Total & 5914 & $58 \cdot 4$ & 1076 & $79 \cdot 6$ & 972 & $88 \cdot 1$ \\
\hline
\end{tabular}

${ }^{*}$ All of the variables included missing data.

+Number measured for waist and hip in 2006 as a percentage of those studied at each follow-up (allowing for selection of census tracts in 1997). $\ddagger \chi^{2}$ test for heterogeneity.

The independent variables were collected at the different follow-up visits (Fig. 1). Self-reported skin colour was collected in 2004 in five groups according to the classification of the Brazilian Institute of Geography and Statistics (white, black, brown, yellow and indigenous) ${ }^{(34)}$. In Brazil, self-reported skin colour is widely used as a proxy for ethnic background. This variable was categorized as white, black/brown or other. The last group included twenty-five individuals who described themselves as 'yellow' or 'indigenous'. Due to the small number of individuals in this group resulting in imprecise estimates, they are not presented as a separate category in the analyses, but they were retained in adjusted analyses to avoid reducing the study power.

The main SEP indicator used in the analyses was family income collected in 1982 and 2004. This variable reflects access to essential resources, including food, quality education and health care ${ }^{(35)}$. In 1982, $21.9 \%$ of all families earned up to one minimum wage (\$US 50/ month) which places them well below the poverty line. In 2004, the incomes of all family members were summed, including the cohort member if currently employed, and only $5.7 \%$ of all families had an income up to one minimum wage (\$US $180 /$ month). To ensure comparability between both periods the variable was divided into tertiles. Details on how the income variables were collected and categorized are available elsewhere ${ }^{(17)}$. Family income change was classified as: (i) always poor (bottom tertile of family income at birth and at age 23-24 years); (ii) never poor (top two tertiles in both periods); (iii) poor-not poor (bottom tertile at birth and top two tertiles at age 23-24 years); and (iv) not poor-poor (top two tertiles at birth and bottom tertile at age 23-24 years). All analyses were stratified by sex.

Own education, behavioural variables and parity (women) were collected in 2004. Dichotomous variables included current smoking ( $\geq 1$ cigarette/d), sedentary behaviour (moderate physical activity $<150 \mathrm{~min} /$ week) and low fibre intake. Ordinal variables were used to describe fat intake (very low, low, American diet, high, very high), alcohol consumption (non-drinker, up to $1 \mathrm{unit} / \mathrm{d},>1 \mathrm{unit} / \mathrm{d}$ ), attained education of the individual $(\leq 7,8-11, \geq 12$ years $)$ and parity $(0,1,2, \geq 3)$. Details on the collection and classification of these variables are available elsewhere ${ }^{(36)}$.

The adjusted analyses took into account the different levels of determination. The most distal determinants were skin colour and family income at birth, which were adjusted for one another (model 1). The next level included family income in 2004, that was adjusted for skin colour and income at birth (model 2). The family income change variable was adjusted solely for skin colour. To investigate possible mediating effects, all of the above variables were adjusted for current behavioural variables and attained education of the individual (model 3). Finally, these analyses were repeated with additional 
adjustment for concurrent BMI (model 4) to assess the effect of each explanatory variable on WC, HC and WHR independently of overall fatness. Variables were dropped from the model when their $P$ value was $\geq 0 \cdot 20$.

ANOVA was used in crude analyses and multiple linear regression in adjusted analyses. Regression coefficients $(\beta)$ and standard errors of the differences relative to the reference category (SED) are expressed in centimetres for WC and $\mathrm{HC}$ and as a percentage for WHR $(\beta$ or SED $\times 100)$. Tests for linear trend were used for ordinal variables. The STATA statistical software package version 9.0 (Statacorp, College Station, TX, USA) was used for analysis.

A posteriori calculations of statistical power showed that the family income change variable had the least power. In men, a difference of means between any two categories equal to or greater than $4.0 \mathrm{~cm}$ in WC, $3.4 \mathrm{~cm}$ for $\mathrm{HC}$ and $2.0 \%$ for WHR was detectable with $80 \%$ power. Corresponding values for women were $3.8 \mathrm{~cm}$, $3.9 \mathrm{~cm}$ and $2.0 \%$. For all other explanatory variables, detectable differences were smaller than these.

The Federal University of Pelotas Ethical Committee approved all phases of the 1982 Pelotas birth cohort study. Verbal informed consent was obtained in 1982 and written informed consent was collected in more recent follow-ups.

\section{Results}

In 2006, 856 individuals had their waist and hip measurements taken $(58 \cdot 4 \%$ of the original cohort; $79 \cdot 6 \%$ of those interviewed in 1997). The sample had higher family income at birth compared with the original cohort, but there were no differences with respect to sex, skin colour, maternal education or birth weight (Table 1). Pregnant and postpartum women at the time of the measurements were excluded ( $n$ 24).

Among men, mean values were $23.8 \mathrm{~kg} / \mathrm{m}^{2}$ for BMI, $82.0 \mathrm{~cm}$ for WC, $97 \cdot 6 \mathrm{~cm}$ for HC and 0.84 for WHR. Corresponding values for women were $23 \cdot 4 \mathrm{~kg} / \mathrm{m}^{2}, 74 \cdot 2 \mathrm{~cm}$, $98.7 \mathrm{~cm}$ and $0 \cdot 75$. All variables were symmetrically distributed. Elevated WC ( $\geq 94 \mathrm{~cm}$ and $\geq 80 \mathrm{~cm}$, respectively) was found in $12 \cdot 0 \%$ of men and in $8 \cdot 2 \%$ of women ${ }^{(3)}$.

Table 2 shows the crude associations between the explanatory variables and WC, HC and WHR. All associations varied according to sex $(P$ for interaction $\leq 0.05$ for all). In men, there were no significant differences in WC or HC according to skin colour. Family income at birth and in adulthood were directly associated with WC and HC. Both measures were highest among men who were never poor and lowest among those who were always poor. None of the explanatory variables were associated with WHR.

In women, WC and WHR were higher in black/brown compared with whites, but HC did not vary by skin colour. Family income in 1982 and 2004 were inversely associated with WC and WHR, but not with HC. Women in the always poor and in the not poor-poor groups had greater WC compared with the other groups ( $P$ of the overall difference $=0 \cdot 06$ ). Women who were poor at birth had lower HC, especially in the poor-not poor group $(P=0.05)$. Women who were always poor had higher WHR than those who were never poor.

Table 3 shows the adjusted analyses for men. Skin colour was not associated with any of the outcomes. The direct associations between family income at birth and both circumferences persisted when adjusted for skin colour (model 1). Further adjustment for family income in 2004 reduced the differences but the associations remained significant (model 2). The coefficients were little affected when adjusted for behavioural variables (model 3). Further adjustment for BMI (model 4) led to a reduction in the coefficients and their standard errors, but the association between early income and both circumferences remained highly significant. WHR was not associated with family income at birth in any level.

Family income in 2004 remained associated with an increase of approximately $4 \mathrm{~cm}$ in both circumferences when adjusted for skin colour and family income at birth (model 2). When behavioural variables were included, these differences fell for HC but not for WC, and both associations remained significant (model 3). The association was made null with further adjustment for BMI (model 4). For WHR, there was no dose-response association with family income in 2004; only model 3 was significant, but the relevance of this association is unclear.

For family income change both circumferences were about $5 \mathrm{~cm}$ larger for men who were never poor compared with those always poor, even after adjustment for skin colour and behavioural variables. There was a substantial reduction after adjustment for BMI, but the association remained significant. WHR was slightly higher among men who were never poor compared with the poor-not poor, but only after adjustment for behavioural variables (model 3); the relevance of this finding is unclear.

Summing up the results for men, both past and concurrent family income were positively associated with WC and HC. These associations were partially mediated by behavioural variables. BMI partially explained the effects of family income at birth and fully explained the effect of the concurrent family income. Whereas both circumferences increased proportionally to income, WHR remained essentially constant. Skin colour showed no associations with the outcome variables.

Table 4 shows the multivariate analyses for women. After adjustment for family income at birth (model 1), WC and $\mathrm{HC}$ were higher in black women compared with white women. The associations remained significant after adjustment for concurrent family income (model 2) and behavioural variables (model 3), but not when current BMI was included (model 4). The association between skin colour and WHR disappeared with adjustment for family income at birth (model 1). 


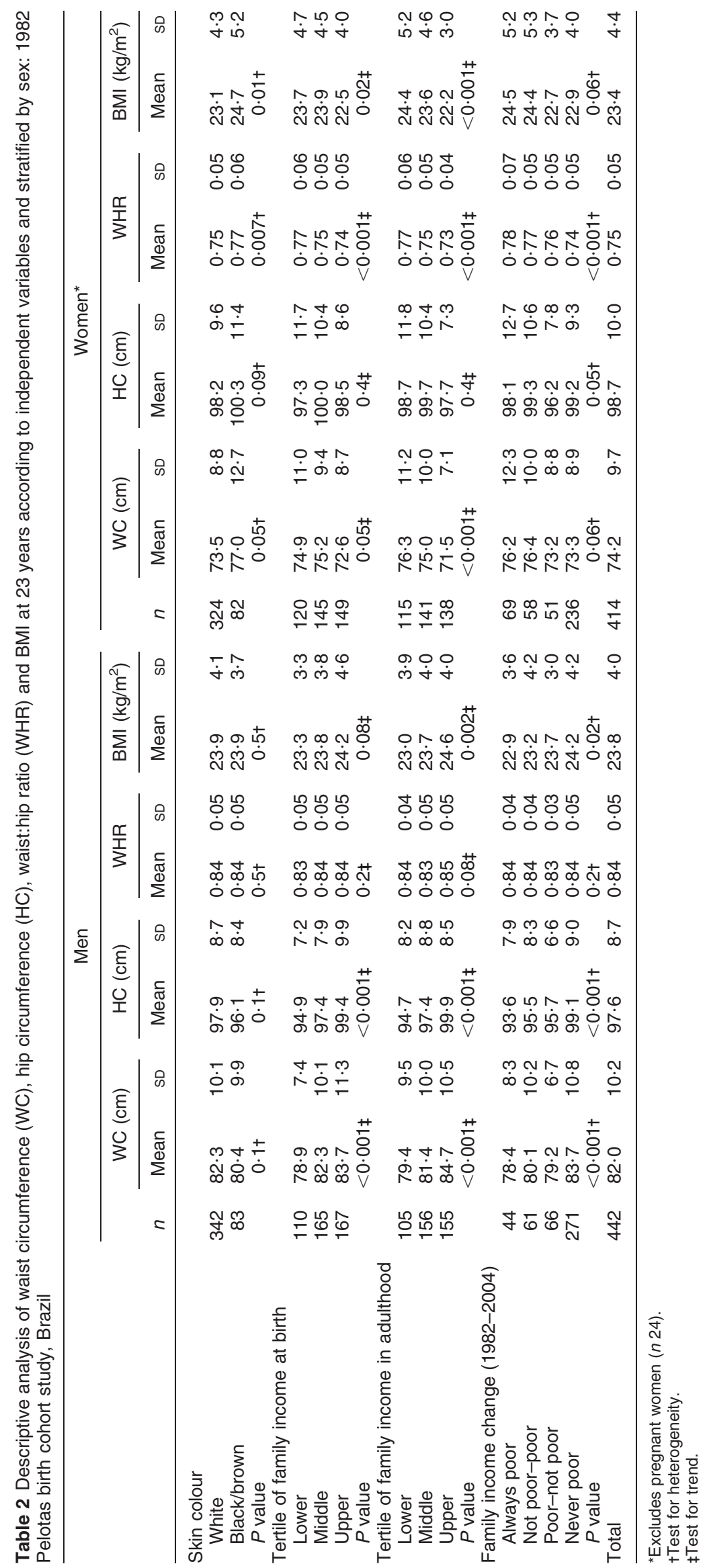


Table 3 Unadjusted and adjusted analyses for waist circumference (WC), hip circumference (HC) and waist:hip ratio (WHR) at 23 years in men according to past and concurrent socio-economic variables: 1982 Pelotas birth cohort study, Brazil

\begin{tabular}{|c|c|c|c|c|c|c|c|c|c|c|}
\hline & \multicolumn{2}{|l|}{ Crude } & \multicolumn{2}{|c|}{ Model 1} & \multicolumn{2}{|c|}{ Model 2} & \multicolumn{2}{|l|}{ Model 3} & \multicolumn{2}{|l|}{ Model 4} \\
\hline & $\beta$ & SED & $\beta$ & SED & $\beta$ & SED & $\beta$ & SED & $\beta$ & SED \\
\hline \multicolumn{11}{|l|}{ WC $(\mathrm{cm})$} \\
\hline Skin colour & $P=0 \cdot 1^{*}$ & & $P=0.9^{*}$ & & $P=0 \cdot 7^{\star}$ & & $P=0 \cdot 7^{*}$ & & $P=0 \cdot 2^{*}$ & \\
\hline White & 0.0 & - & $0 \cdot 0^{\mathrm{a}}$ & - & $0 \cdot 0^{\mathrm{a}, \mathrm{c}}$ & - & $0 \cdot 0^{\mathrm{a}, \mathrm{c}, \mathrm{d}}$ & - & $0 \cdot 0^{\mathrm{a}, \mathrm{c}, \mathrm{d}, \mathrm{e}}$ & - \\
\hline Black/brown & $-1 \cdot 8$ & $1 \cdot 2$ & $0 \cdot 2$ & $1 \cdot 3$ & 0.5 & $1 \cdot 4$ & 0.5 & $1 \cdot 4$ & -0.9 & 0.7 \\
\hline Tertile of family income at birth & $P<0.001 \dagger$ & & $P<0.001 \dagger$ & & $P=0.02 t$ & & $P=0.01 \dagger$ & & $P=0.002 t$ & \\
\hline Lower & 0.0 & - & $0 \cdot 0^{\mathrm{b}}$ & - & $0.0^{\mathrm{b}, \mathrm{c}}$ & - & $0 \cdot 0^{\mathrm{b}, \mathrm{c}, \mathrm{d}}$ & - & $0 \cdot 0^{\mathrm{b}, \mathrm{c}, \mathrm{d}, \mathrm{e}}$ & - \\
\hline Middle & $3 \cdot 4$ & $1 \cdot 2$ & $3 \cdot 7$ & $1 \cdot 3$ & $2 \cdot 9$ & $1 \cdot 4$ & $3 \cdot 0$ & $1 \cdot 4$ & $1 \cdot 8$ & 0.7 \\
\hline Upper & $4 \cdot 8$ & $1 \cdot 2$ & $5 \cdot 2$ & $1 \cdot 3$ & $3 \cdot 7$ & 1.5 & $4 \cdot 2$ & $1 \cdot 6$ & $2 \cdot 5$ & 0.8 \\
\hline Tertile of family income in adulthood & $P<0.001+$ & & & & $P=0.002 t$ & & $P=0.003 t$ & & $P=0 \cdot 1 \dagger$ & \\
\hline Lower & 0.0 & - & & & $0.0^{a, b}$ & - & $0 \cdot 0^{\mathrm{a}, \mathrm{b}, \mathrm{d}}$ & - & $0 \cdot 0^{\mathrm{a}, \mathrm{b}, \mathrm{d}, \mathrm{e}}$ & - \\
\hline Middle & $2 \cdot 0$ & $1 \cdot 3$ & & & 1.5 & $1 \cdot 3$ & $1 \cdot 3$ & $1 \cdot 3$ & $0 \cdot 1$ & 0.6 \\
\hline Upper & $5 \cdot 3$ & $1 \cdot 3$ & & & $4 \cdot 2$ & $1 \cdot 4$ & $4 \cdot 3$ & $1 \cdot 4$ & $1 \cdot 1$ & 0.7 \\
\hline Family income change (1982-2004) & $P<0.001^{*}$ & & & & $P<0.001^{*}$ & & $P=0.002^{*}$ & & $P=0.001^{*}$ & \\
\hline Always poor & $0 \cdot 0$ & - & & & $0 \cdot 0^{\mathrm{b}}$ & - & $0.0^{\mathrm{b}, \mathrm{d}}$ & - & $0 \cdot 0^{b, d, e}$ & - \\
\hline Not poor-poor & $1 \cdot 7$ & $2 \cdot 0$ & & & $2 \cdot 0$ & $2 \cdot 0$ & $2 \cdot 1$ & $2 \cdot 1$ & $0 \cdot 7$ & $1 \cdot 0$ \\
\hline Poor-not poor & $0 \cdot 8$ & 1.9 & & & $0 \cdot 7$ & 1.9 & $1 \cdot 1$ & $2 \cdot 1$ & -0.8 & $1 \cdot 0$ \\
\hline Never poor & $5 \cdot 3$ & $1 \cdot 6$ & & & $5 \cdot 5$ & $1 \cdot 7$ & $5 \cdot 5$ & $1 \cdot 9$ & $2 \cdot 0$ & 0.9 \\
\hline \multicolumn{11}{|l|}{$\mathrm{HC}(\mathrm{cm})$} \\
\hline Skin colour & $P=0 \cdot 1^{*}$ & & $P=1 \cdot 0^{*}$ & & $P=0.9^{*}$ & & $P=0 \cdot 6^{*}$ & & $P=0.3^{*}$ & \\
\hline White & 0.0 & - & $0 \cdot 0^{\mathrm{a}}$ & - & $0 \cdot 0^{\mathrm{a}, \mathrm{c}}$ & - & $0 \cdot 0^{\mathrm{a}, \mathrm{c}, \mathrm{d}}$ & - & $0 \cdot 0^{\mathrm{a}, \mathrm{c}, \mathrm{d}, \mathrm{e}}$ & - \\
\hline Black/brown & $-1 \cdot 8$ & $1 \cdot 1$ & 0.0 & $1 \cdot 1$ & 0.2 & $1 \cdot 2$ & $0 \cdot 4$ & $1 \cdot 2$ & $-0 \cdot 7$ & 0.6 \\
\hline Tertile of family income at birth & $P<0.001+$ & & $P<0.001 \dagger$ & & $P=0.007 \dagger$ & & $P=0.01 t$ & & $P=0.006 t$ & \\
\hline Lower & 0.0 & - & $0.0^{\mathrm{b}}$ & - & $0.0^{\mathrm{b}, \mathrm{c}}$ & - & $0 \cdot 0^{\mathrm{b}, \mathrm{c}, \mathrm{d}}$ & - & $0 \cdot 0^{\mathrm{b}, \mathrm{c}, \mathrm{d}, \mathrm{e}}$ & - \\
\hline Middle & $2 \cdot 6$ & $1 \cdot 1$ & $2 \cdot 7$ & $1 \cdot 1$ & $1 \cdot 9$ & $1 \cdot 2$ & 1.5 & $1 \cdot 2$ & 0.6 & 0.6 \\
\hline Upper & $4 \cdot 6$ & $1 \cdot 1$ & $4 \cdot 7$ & $1 \cdot 2$ & $3 \cdot 4$ & $1 \cdot 3$ & $3 \cdot 3$ & $1 \cdot 3$ & $1 \cdot 9$ & 0.7 \\
\hline Tertile of family income in adulthood & $P<0.001+$ & & & & $P=0.001 \dagger$ & & $P=0.005 t$ & & $P=0.2 \dagger$ & \\
\hline Lower & $0 \cdot 0$ & - & & & $0 \cdot 0^{\mathrm{a}, \mathrm{b}}$ & - & $0 \cdot 0^{\mathrm{a}, \mathrm{b}, \mathrm{d}}$ & - & $0 \cdot 0^{\mathrm{a}, \mathrm{b}, \mathrm{d}, \mathrm{e}}$ & - \\
\hline Middle & $2 \cdot 8$ & $1 \cdot 1$ & & & $2 \cdot 4$ & $1 \cdot 1$ & $2 \cdot 0$ & $1 \cdot 1$ & $1 \cdot 0$ & 0.6 \\
\hline Upper & $5 \cdot 2$ & $1 \cdot 1$ & & & $4 \cdot 1$ & $1 \cdot 2$ & $3 \cdot 5$ & $1 \cdot 2$ & 0.9 & 0.7 \\
\hline Family income change (1982-2004) & $P<0.001^{*}$ & & & & $P<0.001^{*}$ & & $P=0.005^{\star}$ & & $P=0.04^{\star}$ & \\
\hline Always poor & 0.0 & - & & & $0.0^{\mathrm{b}}$ & - & $0 \cdot 0^{\mathrm{b}, \mathrm{d}}$ & - & $0 \cdot 0^{\mathrm{b}, \mathrm{d}, \mathrm{e}}$ & _- \\
\hline Not poor-poor & $1 \cdot 9$ & $1 \cdot 7$ & & & $2 \cdot 0$ & $1 \cdot 7$ & $1 \cdot 4$ & $1 \cdot 8$ & 0.3 & $1 \cdot 0$ \\
\hline Poor-not poor & $2 \cdot 1$ & $1 \cdot 7$ & & & $2 \cdot 1$ & $1 \cdot 7$ & $1 \cdot 9$ & $1 \cdot 7$ & $0 \cdot 3$ & 0.9 \\
\hline Never poor & $5 \cdot 5$ & $1 \cdot 4$ & & & $5 \cdot 6$ & $1 \cdot 5$ & $4 \cdot 5$ & $1 \cdot 6$ & $1 \cdot 7$ & 0.9 \\
\hline \multicolumn{11}{|l|}{ WHR (\%) } \\
\hline Skin colour & $P=0.5^{\star}$ & & $P=0.8^{*}$ & & $P=0.9^{*}$ & & $P=0 \cdot 9^{*}$ & & $P=0.5^{\star}$ & \\
\hline White & $0 \cdot 0$ & - & $0 \cdot 0^{\mathrm{a}}$ & - & $0 \cdot 0^{\mathrm{a}, \mathrm{c}}$ & - & $0 \cdot 0^{\mathrm{a}, \mathrm{c}, \mathrm{d}}$ & - & $0 \cdot 0^{\mathrm{a}, \mathrm{c}, \mathrm{d}, \mathrm{e}}$ & - \\
\hline Black/brown & $-0 \cdot 4$ & 0.6 & $0 \cdot 1$ & 0.6 & 0.2 & 0.6 & $0 \cdot 1$ & 0.7 & -0.4 & 0.5 \\
\hline Tertile of family income at birth & $P=0.2 t$ & & $P=0.2+$ & & $P=0.5 t$ & & $P=0 \cdot 1 \dagger$ & & $P=0.2 \dagger$ & \\
\hline Lower & 0.0 & - & 0.0 & - & $0.0^{\mathrm{c}}$ & - & $0 \cdot 0^{\mathrm{c}, \mathrm{d}}$ & - & $0 \cdot 0^{\mathrm{c}, \mathrm{d}, \mathrm{e}}$ & - \\
\hline Middle & $1 \cdot 2$ & $0 \cdot 6$ & $1 \cdot 2$ & 0.6 & $1 \cdot 0$ & 0.6 & $1 \cdot 4$ & $0 \cdot 6$ & $1 \cdot 3$ & 0.5 \\
\hline Upper & $0 . \overline{9}$ & 0.6 & 0.9 & 0.6 & $0 \cdot 4$ & $0 \cdot 7$ & $1 \cdot 1$ & $0 \cdot 7$ & 0.8 & 0.6 \\
\hline Tertile of family income in adulthood & $P=0.09 t$ & & & & $P=0.2 \dagger$ & & $P=0.02 t$ & & $P=0.4 t$ & \\
\hline Lower & 0.0 & - & & & $0.0^{\mathrm{a}}$ & - & $0 \cdot 0^{\mathrm{a}, \mathrm{d}}$ & - & $0 \cdot 0^{\mathrm{a}, \mathrm{d}, \mathrm{e}}$ & - \\
\hline Middle & $-0 \cdot 3$ & 0.6 & & & $-0 \cdot 4$ & 0.6 & $-0 \cdot 2$ & $0 \cdot 6$ & -0.5 & 0.5 \\
\hline Upper & 0.9 & 0.6 & & & $0 \cdot 8$ & 0.6 & $1 \cdot 4$ & $0 \cdot 7$ & 0.5 & 0.5 \\
\hline Family income change (1982-2004) & $P=0 \cdot 1^{*}$ & & & & $P=0 \cdot 1^{\star}$ & & $P=0.04^{*}$ & & $P=0.06^{*}$ & \\
\hline Always poor & $0 \cdot 0$ & - & & & $0 \cdot 0$ & - & $0 \cdot 0^{\mathrm{d}}$ & - & $0 \cdot 0^{\mathrm{d}, \mathrm{e}}$ & - \\
\hline Not poor-poor & -0.0 & 0.9 & & & $-0 \cdot 0$ & 0.9 & 0.6 & 0.9 & 0.4 & 0.8 \\
\hline Poor-not poor & $-1 \cdot 0$ & 0.9 & & & $-1 \cdot 0$ & 0.9 & $-0 \cdot 4$ & 0.9 & $-1 \cdot 0$ & 0.8 \\
\hline Never poor & 0.5 & $0 \cdot 7$ & & & 0.5 & 0.7 & $1 \cdot 4$ & 0.8 & 0.5 & 0.7 \\
\hline
\end{tabular}

$\beta$, regression coefficient; SED, standard error of the difference.

Adjusted for: ${ }^{a}$ family income at birth; ${ }^{b}$ skin colour; ${ }^{c}$ family income in adulthood; ${ }^{d}$ own education, physical inactivity, alcohol consumption, smoking, fibre and fat intake; ${ }^{\mathrm{e}}$ current BMI.

*Wald test for heterogeneity.

tWald test for trend.

The inverse association between family income at birth and WC lost significance after adjustment for skin colour (model 1). In all four models, HC was larger in the top two tertiles of family income at birth than in the lowest tertile. For WHR, the inverse association with family income at birth remained significant when adjusted for skin colour (model 1); and the association became progressively weaker when adjusted for mediating variables (models 2 to 4 ).

The inverse association between family income in 2004 and WC showed little change in models 2 and 3, but disappeared after adjustment for BMI (model 4). 
Table 4 Unadjusted and adjusted analyses for waist circumference (WC), hip circumference (HC) and waist:hip ratio (WHR) at 23 years in women according to past and concurrent socio-economic variables: 1982 Pelotas birth cohort study, Brazil

\begin{tabular}{|c|c|c|c|c|c|c|c|c|c|c|}
\hline & \multicolumn{2}{|l|}{ Crude } & \multicolumn{2}{|c|}{ Model 1} & \multicolumn{2}{|c|}{ Model 2} & \multicolumn{2}{|l|}{ Model 3} & \multicolumn{2}{|l|}{ Model 4} \\
\hline & $\beta$ & SED & $\beta$ & SED & $\beta$ & SED & $\beta$ & SED & $\beta$ & SED \\
\hline \multicolumn{11}{|l|}{$\mathrm{WC}(\mathrm{cm})$} \\
\hline Skin colour & $P=0.003^{*}$ & & $P=0.01^{*}$ & & $P=0.01^{*}$ & & $P=0.01^{*}$ & & $P=0.6^{\star}$ & \\
\hline White & 0.0 & - & $0 \cdot 0^{\mathrm{a}}$ & - & $0 \cdot 0^{\mathrm{a}, \mathrm{c}}$ & - & $0 \cdot 0^{\mathrm{a}, \mathrm{c}, \mathrm{d}}$ & - & $0 \cdot 0^{\mathrm{a}, \mathrm{c}, \mathrm{d}, \mathrm{e}}$ & - \\
\hline Black/brown & $3 \cdot 6$ & $1 \cdot 2$ & $3 \cdot 3$ & $1 \cdot 3$ & $3 \cdot 3$ & $1 \cdot 3$ & $3 \cdot 4$ & $1 \cdot 3$ & $0 \cdot 3$ & 0.7 \\
\hline Tertile of family income at birth & $P=0.05 t$ & & $P=0.4 \dagger$ & & $P=0.5 \dagger$ & & $P=0 \cdot 3+$ & & $P=0.4 \dagger$ & \\
\hline Lower & 0.0 & - & $0 \cdot 0^{\mathrm{b}}$ & - & $0 \cdot 0^{\mathrm{b}, \mathrm{c}}$ & - & $0 \cdot 0^{\mathrm{b}, \mathrm{c}, \mathrm{d}}$ & - & $0 \cdot 0^{\mathrm{b}, \mathrm{c}, \mathrm{d}, \mathrm{e}}$ & - \\
\hline Middle & $0 \cdot 3$ & $1 \cdot 2$ & $1 \cdot 2$ & $1 \cdot 4$ & $2 \cdot 3$ & $1 \cdot 3$ & $2 \cdot 5$ & $1 \cdot 3$ & $0 \cdot 2$ & 0.7 \\
\hline Upper & $-2 \cdot 2$ & $1 \cdot 2$ & $-1 \cdot 0$ & $1 \cdot 3$ & $1 \cdot 2$ & $1 \cdot 4$ & $1 \cdot 8$ & 1.5 & 0.6 & 0.8 \\
\hline Tertile of family income in adulthood & $P<0.001 \dagger$ & & & & $P<0.001 \dagger$ & & $P=0.003 t$ & & $P=0.5 t$ & \\
\hline Lower & 0.0 & - & & & $0 \cdot 0^{\mathrm{a}, \mathrm{b}}$ & - & $0 \cdot 0^{\mathrm{a}, \mathrm{b}, \mathrm{d}}$ & - & $0 \cdot 0^{\mathrm{a}, \mathrm{b}, \mathrm{d}, \mathrm{e}}$ & - \\
\hline Middle & $-1 \cdot 3$ & $1 \cdot 2$ & & & $-1 \cdot 5$ & $1 \cdot 2$ & $-1 \cdot 6$ & $1 \cdot 3$ & $0 \cdot 6$ & 0.6 \\
\hline Upper & $-4 \cdot 8$ & $1 \cdot 2$ & & & $-4 \cdot 7$ & $1 \cdot 2$ & $-4 \cdot 3$ & 1.5 & -0.5 & 0.7 \\
\hline Family income change (1982-2004) & $P=0.03^{*}$ & & & & $P=0.07^{*}$ & & $P=0 \cdot 1^{*}$ & & $P=0.9^{\star}$ & \\
\hline Always poor & 0.0 & - & & & $0.0^{\mathrm{b}}$ & - & $0 \cdot 0^{\mathrm{b}, \mathrm{d}}$ & - & $0 \cdot 0^{\mathrm{b}, \mathrm{d}, \mathrm{e}}$ & - \\
\hline Not poor-poor & $0 \cdot 3$ & $1 \cdot 7$ & & & 1.5 & $1 \cdot 8$ & $2 \cdot 1$ & $1 \cdot 8$ & 0.6 & 0.9 \\
\hline Poor-not poor & $-3 \cdot 0$ & $1 \cdot 8$ & & & $-2 \cdot 6$ & $1 \cdot 8$ & $-2 \cdot 3$ & 1.9 & $0 \cdot 7$ & $1 \cdot 0$ \\
\hline Never poor & $-2 \cdot 9$ & $1 \cdot 3$ & & & $-1 \cdot 6$ & $1 \cdot 4$ & $-0 \cdot 4$ & $1 \cdot 6$ & 0.6 & 0.8 \\
\hline \multicolumn{11}{|l|}{$\mathrm{HC}(\mathrm{cm})$} \\
\hline Skin colour & $P=0.09^{*}$ & & $P=0.02^{*}$ & & $P=0.02^{*}$ & & $P=0.006^{*}$ & & $P=0.4^{*}$ & \\
\hline White & 0.0 & - & $0 \cdot 0^{a}$ & - & $0 \cdot 0^{\mathrm{a}, \mathrm{c}}$ & - & $0 \cdot 0^{\mathrm{a}, \mathrm{c}, \mathrm{d}}$ & - & $0 \cdot 0^{\mathrm{a}, \mathrm{c}, \mathrm{d}, \mathrm{e}}$ & - \\
\hline Black/brown & $2 \cdot 1$ & $1 \cdot 2$ & $3 \cdot 2$ & $1 \cdot 3$ & $3 \cdot 2$ & $1 \cdot 3$ & $3 \cdot 7$ & $1 \cdot 3$ & $0 \cdot 7$ & 0.8 \\
\hline Tertile of family income at birth & $P=0.4 t$ & & $P=0.09 t$ & & $P=0.04 \dagger$ & & $P=0.04 \dagger$ & & $P=0.01 \dagger$ & \\
\hline Lower & 0.0 & - & $0.0^{\mathrm{b}}$ & - & $0.0^{\mathrm{b}, \mathrm{c}}$ & - & $0.0^{\mathrm{b}, \mathrm{c}, \mathrm{d}}$ & - & $0 \cdot 0^{\mathrm{b}, \mathrm{c}, \mathrm{d}, \mathrm{e}}$ & - \\
\hline Middle & $2 \cdot 6$ & $1 \cdot 2$ & $3 \cdot 4$ & $1 \cdot 3$ & $3 \cdot 6$ & 1.5 & $3 \cdot 6$ & $1 \cdot 4$ & $1 \cdot 4$ & 0.7 \\
\hline Upper & $1 \cdot 3$ & $1 \cdot 2$ & $2 \cdot 5$ & $1 \cdot 3$ & $3 \cdot 3$ & 1.9 & $3 \cdot 4$ & $1 \cdot 6$ & $2 \cdot 2$ & 0.9 \\
\hline Tertile of family income in adulthood & $P=0.4 \dagger$ & & & & $P=0.2 \dagger$ & & $P=0.08 t$ & & $P=0.2 \dagger$ & \\
\hline Lower & 0.0 & - & & & $0 \cdot 0^{\mathrm{a}, \mathrm{b}}$ & - & $0 \cdot 0^{a, b, d}$ & - & $0 \cdot 0^{\mathrm{a}, \mathrm{b}, \mathrm{d}, \mathrm{e}}$ & - \\
\hline Middle & $1 \cdot 0$ & $1 \cdot 2$ & & & $0 \cdot 4$ & $1 \cdot 3$ & -0.5 & $1 \cdot 3$ & $1 \cdot 6$ & 0.7 \\
\hline Upper & $-1 \cdot 0$ & $1 \cdot 2$ & & & -1.9 & $1 \cdot 4$ & $-2 \cdot 6$ & 1.5 & $1 \cdot 2$ & 0.8 \\
\hline Family income change (1982-2004) & $P=0.2^{*}$ & & & & $P=0.06^{*}$ & & $P=0.06^{*}$ & & $P=0.007^{\star}$ & \\
\hline Always poor & 0.0 & - & & & $0 \cdot 0^{\mathrm{b}}$ & - & $0 \cdot 0^{\mathrm{b}, \mathrm{d}}$ & _- & $0 \cdot 0^{\mathrm{b}, \mathrm{d}, \mathrm{e}}$ & _- \\
\hline Not poor-poor & $1 \cdot 2$ & $1 \cdot 8$ & & & $2 \cdot 4$ & $1 \cdot 8$ & $2 \cdot 7$ & 1.9 & $1 \cdot 3$ & $1 \cdot 0$ \\
\hline Poor-not poor & -1.9 & $1 \cdot 8$ & & & $-1 \cdot 6$ & $1 \cdot 8$ & $-1 \cdot 9$ & 1.9 & $1 \cdot 1$ & $1 \cdot 1$ \\
\hline Never poor & $1 \cdot 1$ & $1 \cdot 4$ & & & $2 \cdot 3$ & $1 \cdot 4$ & 1.9 & $1 \cdot 7$ & $2 \cdot 9$ & 0.9 \\
\hline \multicolumn{11}{|l|}{ WHR (\%) } \\
\hline Skin colour & $P=0.007^{\star}$ & & $P=0.4^{*}$ & & $P=0 \cdot 4^{*}$ & & $P=0.7^{\star}$ & & $P=0.4^{*}$ & \\
\hline White & 0.0 & - & $0 \cdot 0^{\mathrm{a}}$ & - & $0 \cdot 0^{\mathrm{a}, \mathrm{c}}$ & - & $0 \cdot 0^{a, c, d}$ & - & $0 \cdot 0^{\mathrm{a}, \mathrm{c}, \mathrm{d}, \mathrm{e}}$ & - \\
\hline Black/brown & $1 \cdot 8$ & 0.6 & $0 \cdot 6$ & $0 \cdot 7$ & $0 \cdot 6$ & $0 \cdot 7$ & $0 \cdot 2$ & $0 \cdot 7$ & -0.5 & 0.6 \\
\hline Tertile of family income at birth & $P<0.001 \dagger$ & & $P<0.001 \dagger$ & & $P=0.06 t$ & & $P=0.2 t$ & & $P=0.1 \dagger$ & \\
\hline Lower & 0.0 & - & $0.0^{\mathrm{b}}$ & - & $0 \cdot 0^{\mathrm{b}, \mathrm{c}}$ & - & $0 \cdot 0^{b, c, d}$ & - & $0 \cdot 0^{\mathrm{b}, \mathrm{c}, \mathrm{d}, \mathrm{e}}$ & - \\
\hline Middle & $-1 \cdot 7$ & 0.6 & $-1 \cdot 5$ & $0 \cdot 7$ & -0.5 & $0 \cdot 7$ & -0.4 & $0 \cdot 7$ & -0.9 & 0.6 \\
\hline Upper & $-3 \cdot 3$ & 0.6 & $-3 \cdot 0$ & $0 \cdot 7$ & $-1 \cdot 4$ & $0 \cdot 7$ & -0.9 & 0.8 & $-1 \cdot 1$ & 0.7 \\
\hline Tertile of family income in adulthood & $P<0.001 \dagger$ & & & & $P<0.001 \dagger$ & & $P=0.002 t$ & & $P=0.04 \dagger$ & \\
\hline Lower & 0.0 & - & & & $0.0^{\mathrm{a}, \mathrm{b}}$ & - & $0 \cdot 0^{\mathrm{a}, \mathrm{b}, \mathrm{d}}$ & - & $0 \cdot 0^{\mathrm{a}, \mathrm{b}, \mathrm{d}, \mathrm{e}}$ & - \\
\hline Middle & $-2 \cdot 1$ & 0.6 & & & $-1 \cdot 9$ & 0.6 & $-1 \cdot 3$ & $0 \cdot 7$ & $-0 \cdot 8$ & 0.6 \\
\hline Upper & $-4 \cdot 1$ & 0.6 & & & $-3 \cdot 4$ & $0 \cdot 7$ & $-2 \cdot 4$ & $0 \cdot 8$ & $-1 \cdot 5$ & 0.7 \\
\hline Family income change (1982-2004) & $P<0.001^{*}$ & & & & $P=0.01^{*}$ & & $P=0.02^{*}$ & & $P=0.04^{*}$ & \\
\hline Always poor & 0.0 & - & & & $0 \cdot 0^{\mathrm{b}}$ & - & $0.0^{b, d}$ & - & $0 \cdot 0^{\mathrm{b}, \mathrm{d}, \mathrm{e}}$ & - \\
\hline Not poor-poor & -0.7 & 0.9 & & & -0.5 & 0.9 & $-0 \cdot 1$ & $1 \cdot 0$ & -0.5 & 0.9 \\
\hline Poor-not poor & $-1 \cdot 6$ & 0.9 & & & $-1 \cdot 5$ & 0.9 & $-1 \cdot 1$ & $1 \cdot 0$ & $-0 \cdot 3$ & 0.9 \\
\hline Never poor & $-3 \cdot 8$ & 0.7 & & & $-3 \cdot 5$ & $0 \cdot 7$ & $-2 \cdot 1$ & 0.8 & $-1 \cdot 8$ & 0.8 \\
\hline
\end{tabular}

$\beta$, regression coefficient; SED, standard error of the difference.

Adjusted for: ${ }^{a}$ family income at birth; ${ }^{b}$ skin colour; ${ }^{c}$ family income in adulthood; ${ }^{d}$ own education, physical inactivity, alcohol consumption, smoking, fibre and fat intake; ${ }^{\mathrm{e}}$ current BMI.

*Wald test for heterogeneity.

tWald test for trend.

The association with HC was not significant in any of the models. Given its effect on WC, family income in 2004 was inversely associated with WHR in all analyses.

In terms of family income trajectory, WC tended to be smaller among women who were not poor as adults, but the differences were not quite significant (models 2 and 3). This effect disappeared after adjustment for BMI (model 4). HC was approximately $2-3 \mathrm{~cm}$ larger among women who were not poor at birth (never poor and not poor-poor groups) as shown in models 2 to 4 . As a consequence, women who were never poor had lower WHR than other groups in all the models. 
All of the analyses in women were repeated including parity as a possible mediator, but there were no noteworthy changes in any of the associations. Additional analyses restricted to nulliparous women also showed similar results.

Summarizing the results for women, early poverty was associated with smaller hips and current poverty with larger waists. Poverty at any age, therefore, led to higher WHR. Black women had higher WC and HC than white women, and consequently there were no differences in WHR. Behavioural variables played relatively weak mediating roles, but current BMI seemed to mediate most of the effects of current income.

Maternal (1982) and own education (2004) were also evaluated as independent variables, but they were not associated with the outcomes when adjusted for income and skin colour (results not shown).

\section{Discussion}

The present study aimed to investigate the influence of early and concurrent family income, socio-economic trajectory and skin colour on WC, HC and WHR in young adults. There were three differences between the sexes with respect to SEP. First, family income at birth was directly associated with WC in men, but not in women. Second, WC and HC were larger in men with high current family income, but the opposite was true in women, especially for WC. Finally, family income change had a clear cumulative effect in men (both circumferences were larger among the never poor group) but not in women (HC was directly associated with family income at birth whereas WC was inversely associated with current family income). The only consistent finding in both sexes was the direct association between family income at birth and HC. For skin colour, black/brown women had larger WC and HC than whites, but no differences in WHR were found. In men none of the measurements were related to skin colour.

The long-term effects of early SEP on abdominal obesity may be better understood by considering tissue growth. WC is affected principally by changes in subcutaneous and visceral adipose tissue. On the other hand, $\mathrm{HC}$ can vary according to change in fat, bone and muscle. All these tissues represent distinct periods of growth. Adipogenesis begins in utero and the number of adipocytes is determined during this period ${ }^{(13,37)}$. After birth there are three periods with relatively fast physiological fat mass increases: (i) the first sixth months of life; (ii) during the adiposity rebound (5-6 years); and (iii) puberty $^{(12-14)}$. Hip bone formation is determined in the fetal period via hormonal, nutritional and mechanical factors, the latter two exerting effects until the first year of life ${ }^{(13,38,39)}$. During puberty, hormonal factors (oestrogen) become influential again, determining morphology in both sexes ${ }^{(13,39)}$. Muscle tissue is also formed early in life but changes in muscle volume begin at puberty due to hormonal and nutritional factors and physical activity $^{(12-14)}$. In adulthood fat tissue in the abdomen accumulates progressively, and after 60 years of age a decrease of muscle mass in the hip is found ${ }^{(14,40-42)}$. Consequently, there are critical periods for tissues growth - fetal life, early childhood and puberty - that are susceptible to adverse environmental conditions ${ }^{(43)}$.

In men, our findings are consistent with the observation that the wealthier are more likely to be overweight in middle-income countries ${ }^{(8,15)}$. A possible explanation for the association between family income at birth and WC is that wealth affects early-life fat deposition, with an independent and cumulative effect of adult poverty on abdominal adiposity. The same was observed for HC, which is partly reflective of fat tissue. HC in men was affected by current income independently of early childhood income, suggesting that socio-economic trajectories can also influence hip growth in late childhood and adolescence. Two Brazilian studies found the same direct relationships between SEP in childhood and BMI in 18 - to 19 -year-old men ${ }^{(17,44)}$, but no other studies from low- or middle-income countries were located. Six studies evaluated the association between early SEP and WHR in adults in high-income countries, five showing inverse associations for both sexes ${ }^{(20,21,23-25)}$ and one finding an inverse relationship in women and no association in men ${ }^{(22)}$. Only one study was located from the low- and middle-income countries (China), which reported a direct association in males and an inverse one in females ${ }^{(27)}$.

In women, even in middle-income countries, there is an inverse association between concurrent SEP and obesity in adulthood $^{(7,8,15)}$. Our findings add to the evidence suggesting that concurrent poverty - rather than early SEP - determines central obesity. Poverty in early life, however, is associated with lower HC independent of current SEP, probably due to early developmental effects on the bony or muscle structure of the hip. Adverse conditions in the perinatal period related to poverty (obstetric complications, low maternal height, maternal and/or fetal undernutrition) can affect the anatomy of the hip, as well as cause reductions in bone mass that persist until adult$\operatorname{hood}^{(38,39)}$. Other osseous components can also be affected; for example, early-life poverty was shown to be associated with lower height at 18-19 years in the same cohort $^{(17)}$. These findings agree with a recent review on the long-term effect of maternal/infant nutrition ${ }^{(45)}$.

Associations between SEP and the outcomes tested were partially explained by adult behavioural variables such as alcohol consumption, physical inactivity, poor dietary habits (all directly associated with adiposity) and smoking (inversely associated) ${ }^{(46,47)}$. In Pelotas, alcohol consumption is most common among richer men but there is no pattern among women ${ }^{(48)}$. Smoking, on the other hand, is more common among poorer men and 
women. Moreover, there is a strong inverse association between overall physical activity and income in men ${ }^{(49)}$. In women, levels of activity were slightly higher in the poorest, but due to low-intensity domestic activities. Several authors have suggested that poor women in middle- and high-income countries have nutrient-poor, energy-dense diets ${ }^{(3,7,8,15)}$ and may not be as concerned with the aesthetic aspects of thinness. These differences partly explain the higher frequency of abdominal obesity in richer men and the less intense mediating effects of behavioural variables in women.

Current BMI is closely associated to the outcomes under study, and BMI-adjusted analyses investigate possible specific effects beyond an association with overall fatness. SEP at birth remained associated with WC (in men) and HC (in both sexes) after adjustment for current BMI, suggesting that early-life influences on the growth of bone and muscle, and on fat distribution, lead to permanent effects on body shape. On the other hand, concurrent SEP, while strongly associated with BMI (directly in men and inversely in women), was no longer associated with the outcomes after controlling for BMI. This suggests that - unlike early-life poverty - the effects of adult wealth or poverty are not specifically related to either waist or hip dimensions.

Parity has shown a direct association with BMI in the cohort $^{(50)}$ as well as with WC but not HC. Nevertheless, in the present analysis, adjustment for parity did not remove the social differences.

The associations with skin colour are likely related to socio-economic factors, as whites compose $67 \%$ of the lowest tertile of income and $87 \%$ of the highest. In women, several risk factors for CVD - obesity, hypertension, diabetes and insulin resistance - are more common in blacks than in whites ${ }^{(4,28)}$. This association has also been described with respect to $\mathrm{WHR}^{(18,26,29,30)}$. Croft et $a l^{(26)}$ showed that the difference in WHR between US blacks and whites was reduced but remained significant after adjustment for education and behavioural variables. Our results in women show that racial/ethnic differences in WHR disappeared after adjustment for SEP, but that the effects of the adjustment were different for waist and hip measures. The positive confounding for WC suggests that black women have larger waist due to being poorer and adopting the behavioural habits of this social group. For $\mathrm{HC}$, there was negative confounding, suggesting that the biological growth potential of black women's hips is not being attained due to nutritional restrictions associated with poverty in early life. In men, in accordance with the literature, there were no differences in waist or hip measures according to skin colour ${ }^{(4,18,31)}$.

Finally, associations between WHR and SEP result from combined effects of socio-economic trajectories on WC and HC. Men with higher family income at birth, higher concurrent income and who were never poor had both greater WC and HC, which may explain the lack of association with WHR. In women, WHR was inversely associated with income at birth (due to smaller HC among the poor) and in adult life (due to the inverse association between current income and WC). There were no differences in WHR according to skin colour - black women had both larger waists and hips than white women, whereas there were no differences in either indicator among men.

Our analytical models were possible owing to the longitudinal nature of the study. One methodological limitation is that $\sim 40 \%$ of the original cohort members were not followed up. It is difficult to speculate how more losses among poorer individuals could have affected the analyses described here, but it is unlikely that they led to bias. Another possible limitation is the relatively low frequency of individuals with higher levels of abdominal obesity at 23-24 years. Nevertheless, this did not affect the results and even at this age differences of $3-5 \mathrm{~cm}$ in WC and HC were found between SEP groups. Finally, the ratio of white to black/brown subjects was lower compared with that in most other parts of Brazil. However, our results of higher levels of abdominal obesity among black women compared with whites and on the lack of differences in men are consistent with the literature ${ }^{(4,18,26,28-30)}$.

In conclusion, we found strong sex-specific associations between socio-economic trajectories and the anthropometric measures evaluated. The early effects of poverty may be irreversible and therefore prevention depends on interventions aimed at the first years of life.

\section{Acknowledgements}

Sources of funding: The study was partially funded by The Wellcome Trust (Major Awards for Latin America on Health Consequences of Population Change). The initial phases of the cohort study were made possible by support from the National Program for Centers of Excellence (PRONEX), the Brazilian Ministry of Health (National Health Foundation), the International Development Research Center of Canada, and the United Nations Development Fund for Women (UK). Conflict of interest declaration: No conflicts of interests exist. No portion of the work has been or is currently under consideration for publication elsewhere nor has any other portion been published or posted on the Internet. Being accepted for Public Health Nutrition the paper will not be published elsewhere in the same form, in English or in any other language, without the written consent of the Nutrition Society. Authors' contributions: D.A.G. was responsible for data collection, analysis and drafted the results of the study. A.N. participated in data collection and writing of the article. J.S.Y. contributed to the analyses and writing. C.G.V. supervised the study, participated in the analyses and writing. All of the authors read and approved the final manuscript. Acknowledgements: The authors would 
like to thank the researchers at the Center for Epidemiologic Research at the Federal University of Pelotas. Special thanks to Dr Denise Petrucci Gigante and Dr Anaclaudia Gastal Fassa for contributing data from the 1982 Pelotas Cohort and also to Jeovany Martínez Mesa, María Angélica Barbosa and María del Pilar Velez for their contributions to the data collection.

\section{References}

1. de Koning L, Merchant AT, Pogue J \& Anand SS (2007) Waist circumference and waist-to-hip ratio as predictors of cardiovascular events: meta-regression analysis of prospective studies. Eur Heart J 28, 850-856.

2. Lanas F, Avezum A, Bautista LE, Diaz R, Luna M, Islam S \& Yusuf S (2007) Risk factors for acute myocardial infarction in Latin America: the INTERHEART Latin American study. Circulation 115, 1067-1074.

3. World Health Organization (2000) Obesity: Preventing and Managing the Global Epidemic. Report of a WHO Consultation. WHO Technical Report Series no. 894. Geneva: WHO.

4. Yusuf S, Reddy S, Ounpuu S \& Anand S (2001) Global burden of cardiovascular diseases: Part II: variations in cardiovascular disease by specific ethnic groups and geographic regions and prevention strategies. Circulation 104, 2855-2864.

5. Yusuf S, Hawken S, Ounpuu S et al. (2005) Obesity and the risk of myocardial infarction in 27,000 participants from 52 countries: a case-control study. Lancet 366, 1640-1649.

6. Jorgensen ME, Borch-Johnsen K \& Bjerregaard P (2006) Lifestyle modifies obesity-associated risk of cardiovascular disease in a genetically homogeneous population. $A m \mathrm{~J}$ Clin Nutr 84, 29-36.

7. Monteiro CA, Conde WL \& Popkin BM (2007) Incomespecific trends in obesity in Brazil: 1975-2003. Am J Public Health 97, 1808-1812.

8. Monteiro CA, Moura EC, Conde WL \& Popkin BM (2004) Socioeconomic status and obesity in adult populations of developing countries: a review. Bull World Health Organ 82, 940-946.

9. Iacobellis G \& Sharma AM (2007) Obesity and the heart: redefinition of the relationship. Obes Rev $\mathbf{8}, 35-39$.

10. Seidell JC, Perusse L, Despres JP \& Bouchard C (2001) Waist and hip circumferences have independent and opposite effects on cardiovascular disease risk factors: the Quebec Family Study. Am J Clin Nutr 74, 315-321.

11. Snijder MB, Dekker JM, Visser M, Bouter LM, Stehouwer CD, Yudkin JS, Heine RJ, Nijpels G \& Seidell JC (2004) Trunk fat and leg fat have independent and opposite associations with fasting and postload glucose levels: the Hoorn study. Diabetes Care 27, 372-377.

12. Sinclair D \& Dangerfield P (1998) Human Growth after Birth, 6th ed. New York: Oxford University Press.

13. Tanner JM (1989) Foetus into Man, 2nd ed. Ware: Castlemead Publications.

14. Taylor RW, Grant AM, Goulding A \& Williams SM (2005) Early adiposity rebound: review of papers linking this to subsequent obesity in children and adults. Curr Opin Clin Nutr Metab Care 8, 607-612.

15. McLaren L (2007) Socioeconomic status and obesity. Epidemiol Rev 29, 29-48.

16. Sobal J \& Stunkard AJ (1989) Socioeconomic status and obesity: a review of the literature. Psychol Bull $\mathbf{1 0 5}$, 260-275.

17. Barros AJ, Victora CG, Horta BL, Goncalves HD, Lima RC \& Lynch J (2006) Effects of socioeconomic change from birth to early adulthood on height and overweight. Int $J$ Epidemiol 35, 1233-1238.

18. Dekkers JC, Podolsky RH, Treiber FA, Barbeau P, Gutin B \& Snieder H (2004) Development of general and central obesity from childhood into early adulthood in African American and European American males and females with a family history of cardiovascular disease. Am J Clin Nutr 79, 661-668.

19. Galobardes B, Lynch JW \& Davey Smith G (2004) Childhood socioeconomic circumstances and cause-specific mortality in adulthood: systematic review and interpretation. Epidemiol Rev 26, 7-21.

20. Kivimaki M, Smith GD, Juonala M et al. (2006) Socioeconomic position in childhood and adult cardiovascular risk factors, vascular structure, and function: Cardiovascular Risk in Young Finns Study. Heart 92, 474-480.

21. Kuh D, Hardy R, Chaturvedi N \& Wadsworth ME (2002) Birth weight, childhood growth and abdominal obesity in adult life. Int J Obes Relat Metab Disord 26, 40-47.

22. Laitinen J, Pietilainen K, Wadsworth M, Sovio U \& Jarvelin MR (2004) Predictors of abdominal obesity among 31-y-old men and women born in Northern Finland in 1966. Eur J Clin Nutr 58, 180-190.

23. Laitinen J, Power C \& Jarvelin MR (2001) Family social class, maternal body mass index, childhood body mass index, and age at menarche as predictors of adult obesity. Am J Clin Nutr 74, 287-294.

24. Langenberg C, Hardy R, Kuh D, Brunner E \& Wadsworth M (2003) Central and total obesity in middle aged men and women in relation to lifetime socioeconomic status: evidence from a national birth cohort. $J$ Epidemiol Community Health 57, 816-822.

25. Poulton R, Caspi A, Milne BJ, Thomson WM, Taylor A, Sears MR \& Moffitt TE (2002) Association between children's experience of socioeconomic disadvantage and adult health: a life-course study. Lancet 360, 1640-1645.

26. Croft JB, Freedman DS, Keenan NL, Sheridan DP, Macera CA \& Wheeler FC (1996) Education, health behaviors, and the black-white difference in waist-to-hip ratio. Obes Res $\mathbf{4}$, 505-512.

27. Schooling CM, Jiang CQ, Lam TH, Zhang WS, Cheng KK \& Leung GM (2008) Life-course origins of social inequalities in metabolic risk in the population of a developing country. Am J Epidemiol 167, 419-428.

28. Cossrow N \& Falkner B (2004) Race/ethnic issues in obesity and obesity-related comorbidities. J Clin Endocrinol Metab 89, 2590-2594.

29. Harris MM, Stevens J, Thomas N, Schreiner P \& Folsom AR (2000) Associations of fat distribution and obesity with hypertension in a bi-ethnic population: the ARIC study. Atherosclerosis Risk in Communities Study. Obes Res 8 , 516-524.

30. Hill JO, Sidney S, Lewis CE, Tolan K, Scherzinger AL \& Stamm ER (1999) Racial differences in amounts of visceral adipose tissue in young adults: the CARDIA (Coronary Artery Risk Development in Young Adults) study. Am J Clin Nutr 69, 381-387.

31. Wagner DR \& Heyward VH (2000) Measures of body composition in blacks and whites: a comparative review. Am J Clin Nutr 71, 1392-1402.

32. Instituto Brasileiro de Geografia e Estatística (2002) Produto Interno Bruto a preço de mercado e Produto Interno Bruto per capita, segundo as grandes regiões, unidades da federação e municípios - 1999-2002. Brazil: IBGE; available at http://www.ibge.gov.br/home/estatistica/ economia/pibmunicipios/2002/tab01.pdf

33. Victora CG \& Barros FC (2006) Cohort profile: the 1982 Pelotas (Brazil) birth cohort study. Int J Epidemiol 35, 237-242.

34. Travassos C \& Williams DR (2004) The concept and measurement of race and their relationship to public 
health: a review focused on Brazil and the United States. Cad Saude Publica 20, 660-678.

35. Sobal J (1991) Obesity and socioeconomic status: a framework for examining relationships between physical and social variables. Med Anthropol 13, 231-247.

36. Nazmi A, Oliveira IO \& Victora CG (2008) Correlates of C-reactive protein levels in young adults: a populationbased cohort study of 3827 subjects in Brazil. Braz J Med Biol Res 41, 357-367.

37. Fonseca-Alaniz MH, Takada J, Alonso-Vale MI \& Lima FB (2006) The adipose tissue as a regulatory center of the metabolism. Arq Bras Endocrinol Metabol 50, 216-229.

38. Cooper C, Westlake S, Harvey N, Javaid K, Dennison E \& Hanson M (2006) Review: developmental origins of osteoporotic fracture. Osteoporos Int 17, 337-347.

39. Sizínio S \& Xavier R (2003) Ortopedia e Traumatologia: Princípios e Prâticas, 3rd ed. Porto Alegre: Artmed Editora.

40. Dobbelsteyn CJ, Joffres MR, MacLean DR \& Flowerdew G (2001) A comparative evaluation of waist circumference, waist-to-hip ratio and body mass index as indicators of cardiovascular risk factors. The Canadian Heart Health Surveys. Int J Obes Relat Metab Disord 25, 652-661.

41. Heitmann BL (1992) Obesity and body fat distribution among adult Danes aged 35-65 years. An epidemiological study. Ugeskr Laeger 154, 1252-1257.

42. Zafon C (2007) Oscillations in total body fat content through life: an evolutionary perspective. Obes $\operatorname{Rev} \mathbf{8}, 525-530$.

43. Power C \& Parsons T (2000) Nutritional and other influences in childhood as predictors of adult obesity. Proc Nutr Soc 59, 267-272.
44. Goldani MZ, Haeffner LS, Agranonik M, Barbieri MA, Bettiol H \& Silva AA (2007) Do early life factors influence body mass index in adolescents? Braz J Med Biol Res 40, 1231-1236.

45. Victora CG, Adair L, Fall C, Hallal PC, Martorell R, Richter L \& Sachdev HS (2008) Maternal and child undernutrition: consequences for adult health and human capital. Lancet 371, 340-357.

46. Koh-Banerjee P, Chu NF, Spiegelman D, Rosner B, Colditz G, Willett W \& Rimm E (2003) Prospective study of the association of changes in dietary intake, physical activity, alcohol consumption, and smoking with 9-y gain in waist circumference among 16587 US men. Am J Clin Nutr 78, 719-727.

47. Lahmann PH, Lissner L, Gullberg B \& Berglund G (2000) Sociodemographic factors associated with long-term weight gain, current body fatness and central adiposity in Swedish women. Int $J$ Obes Relat Metab Disord 24 , 685-694.

48. Castanheira M, Olinto MT \& Gigante DP (2003) Socio-demographic and lifestyle factors associated with abdominal fat distribution in adults: a population-based survey in Southern Brazil. Cad Saude Publica 19, Suppl. 1, S55-S65.

49. Hallal PC, Victora CG, Wells JC \& Lima RC (2003) Physical inactivity: prevalence and associated variables in Brazilian adults. Med Sci Sports Exerc 35, 1894-1900.

50. Gigante DP, Rasmussen KM \& Victora CG (2005) Pregnancy increases BMI in adolescents of a population-based birth cohort. J Nutr 135, 74-80. 